\title{
The bactericidal efficacy of cold atmospheric plasma technology on some bacterial strains
}

\author{
Raja M. Moman and Hmeda Najmaldeen* \\ Department of Microbiology and Immunology, Faculty of Pharmacy, AL-Fateh \\ University for Medical Sciences and Plasma Research laboratory. \\ *eldein0@yahoo.com
}

\begin{abstract}
Plasma, a mix of ionized gas molecules and free electrons, is often referred to as the fourth state of matter. There are different applications of plasma in our life starts from easy lighting to disease fighting and it's nothing new. Fluorescent lights, air conditions and plasma televisions use it. One of its different types is atmospheric cold plasma, the possible applications for sterilization using cold plasmas range from the food industry to planetary space missions. The same technique could also be used on space craft leaving Earth to avoid transporting micro-organisms from Earth to other planets or moons.

The use of toxic chemicals to sterilize medical instruments may soon be a thing of the past because the use of cold plasma to sterilize heat-sensitive reusable medical tools in a rapid, safe, and effective way is bound to replace the present method which uses a toxic gas as ethylene oxide, in addition to its use for air purification. Lately it is tested to prepare surfaces for bonding and kill bacteria on delicate living tissues.

We report the results of an interdisciplinary collaboration formed to assess the sterilizing capabilities of the cold atmospheric plasma. This newly-invented source of plasma is capable of operating at atmospheric pressure in air and other gases, and of providing antimicrobial activity at room temperature as judged by viable plate counts. Plasma exposures have reduced log numbers of three tested bacterial strains namely, Staphylococcus aureus, Escherichia coli and Pseudomonas aeruginosa seeded on solid surfaces of Muller-Hinton agar at room temperature. Initial experimental data showed $\geq 5$ $\log _{10}$ CFU reduction of bacteria when $5 \times 10^{6} \mathrm{cfu}^{-\mathrm{ml}^{-1}}$ of samples seeded on MHA plates. Results showed $>5 \log _{10}$ CFU reduction with $E$. coli when exposed for up to $360 \mathrm{sec}$ to plasma while the same exposure time was required for $5 \log _{10}$ CFU reduction killing with $S$. aureus samples, the least affected by this treatment was Pseudomonas aeruginosa cell suspensions where there was a very few reduction in number of survivals $(\leq 10 \%$ of the whole population) after the same exposure time application. For all microorganisms tested, a biphasic curve was generated when the number of survivors versus time was plotted in dose-response curves.

In conclusion we can report that the atmospheric cold plasma generated by this method has proven sterilization (kill) capability against both gram-positive and gramnegative bacteria in different extents depending on special strain characteristics.
\end{abstract}

Keywords: Cold atmospheric plasma, bacterial strains, toxic chemicals

\section{INTRODUCTION}

The scientific control of microbial growth began only about 100 years ago. In the mid 1800 s, the Hungarian physician Ignatz Semmelweis and English physician Joseph Lister developed some of the first microbial control practices for medical procedures. These practices included hand washing with microbe killing chloride of lime and aseptic surgery techniques to prevent microbial contamination of surgical wounds.

Over the last century, scientists have continued to develop a variety of physical methods and chemical agents 
to control microbial growth, Tortora J. G. et al. (2010).

Utilizing an ionized gas (plasma) to achieve sterilization is an alternative to conventional sterilization means as far as sterilization of heat-sensitive materials and innocuity of sterilizing agents are concerned.

In 1928, Langmuir and Tonks, while investigating electric discharges at the General Electric Research Laboratory, introduced the term "plasma" to describe the ionized gas. The plasmas are electrically charged gases, or "ionized" gases, that are found in nature as well as in numerous devices. In addition to surrounding you in the universe, plasmas play key roles in bringing you sunlight outdoors during the daytime, street light at night, fluorescent light in your offices, computer chips in your computers, treated and coated surfaces of more important objects in your life than you might imagine (including hardened artificial joints you might have in your body and high temperature turbine blade coatings for the jets you fly in), flat panel displays for your TV, gas lasers, the welded joints that keep buildings from falling apart, and radio transmission around the world. Among the important applications expected in the future is the production of electrical energy from nuclear fusion, environmental cleanup, Herman, V. B. (1982).

A plasma is a hot ionized gas consisting of approximately equal numbers of positively charged ions and negatively charged electrons. The characteristics of plasmas are significantly different from those of ordinary neutral gases, For example, because plasmas are made up of electrically charged particles, they are strongly influenced by electric and magnetic fields while neutral gases are not.
Plasma applications for sterilization

Plasma sterilization, a new application of low-pressure plasma, is a promising technique and an alternative to other conventional sterilization methods like high temperature sterilization, ethylene oxide sterilization and sterilization by radiation, especially for treatment of heat sensitive materials. It promotes an efficient inactivation of the microorganisms and minimizes damage to the materials. UV photons and reactive species like atoms and radicals play a major role in plasma sterilization.

Pérez-Martínez, J. A. et al. 2007 developed RF normal pressure plasma discharge technologies to cleanse and sterilize dental cavities. Ohkawa et al., 2006 used a mixture of helium and oxygen at atmospheric pressure as a sterilization medium in continuous plasma processing of medical-care materials. It was found that destruction of the cell wall was the major mechanism of disinfection. Various treatment times were found to be effective for various microorganisms. Low temperature radio frequency glow discharge plasma was found to be the most suitable for sterilizing polyester devices in tissue engineering applications. Polymers sterilized by plasma showed little or no change in their 3D morphology, molecular weight or mechanical properties, Kwarteng, K. B. and Stark, C., (1990) and Holy, C. E. et al. (2001).

When cellulose strips that contained Bacilli stearothermophili were directly exposed to plasma, complete inactivation took place after $7 \mathrm{~min}$ of exposure, Lerouge, S. et al. (2000).

It has also been reported that plasma sterilization diminishes platelet deposition without affecting 
the coagulation time, Moreira, A. J. et al. (2004).

Antimicrobial coatings on RF plasma-treated biopolymers can prevent microbial adherence on polymer surface, thus preventing biofilm formation, Stavridi, M. et al. (2003).

\section{EXPERIMENTAL METHODS}

Plasmas for medical settings must be uniform in addition to having low energy. To be useful, they must also remain uniform in air at atmospheric pressur.

The voltages we used, 20 to 30 $\mathrm{kV}$, were enough to overcome the dielectric constant of the air and break it down into plasma.

Voltage generates lightning between two electrodes in our setup, which are separated by an air gap. By covering one electrode with a strong dielectric, we keep the charge from following a direct path between electrodes. Lacking a specific path, the discharge spreads uniformly throughout the intervening space (fig.1). This study was conducted to determine the efficacy of plasma for inactivation of both gram-positive and gram-negative bacteria namely, Escherichia coli, Staphylococcus aureus and Pseudomonas aeruginosa. The strain mixture of each bacterium was inoculated $\left(5 \times 10^{6} \mathrm{cfu} / \mathrm{ml}\right)$ onto a microscope slides containing Muller Hinton agar media then samples exposed to plasma for $0,30,60180$ and 360 seconds.

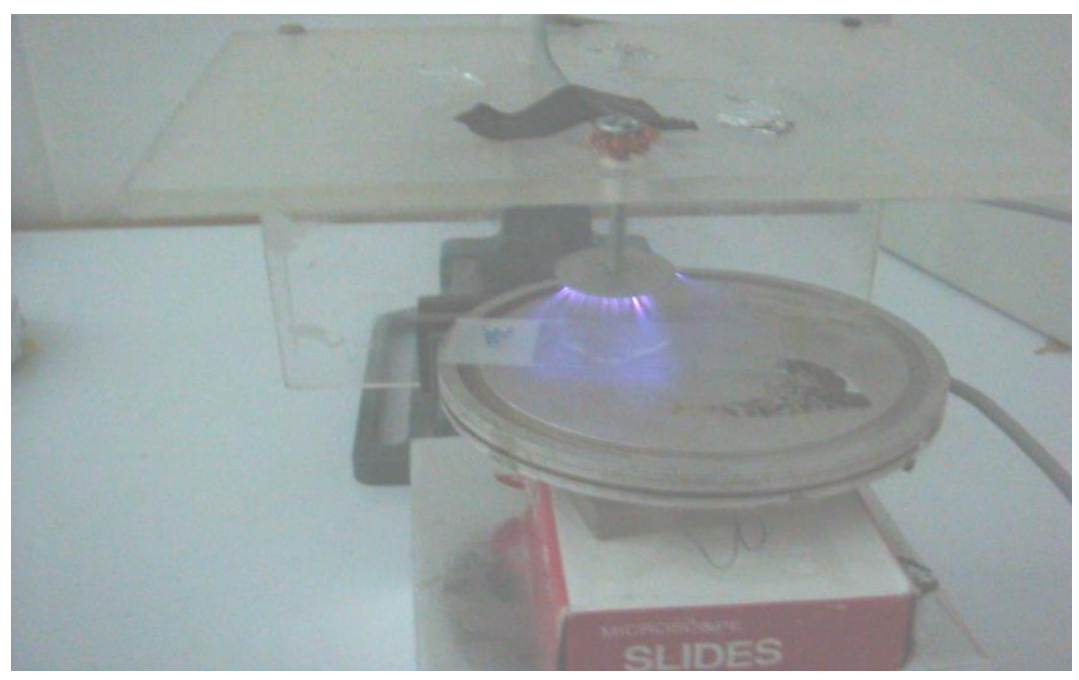

Fig. 1: Generation of cold plasma and samples treatment.

After exposure, the agar removed from the slides and pummeled in $0.1 \%$ peptone water, serially diluted, surface plated onto nonselective media, and incubated at $37^{\circ} \mathrm{C}$ for 24 hours.

\section{RESULTS AND DISCUSSIONS}

Plasma exposures have reduced log numbers of three tested bacterial strains seeded on solid surfaces of Muller-Hinton agar at room temperature. Initial experimental data showed $\geq 5 \log _{10} \mathrm{CFU}$ reduction of bacteria when $5 \times 10^{6}$ cfu.ml $^{-1}$ of samples seeded on MHA plates.

Results showed $>5 \log _{10} \mathrm{CFU}$ reduction with $E$. coli when exposed for up to $360 \mathrm{sec}$ (fig.2) to plasma while the same exposure time was required for $5 \log _{10} \mathrm{CFU}$ reduction killing with $S$. aureus samples, the least affected by this treatment was Pseudomonas aeruginosa cell suspensions where there was a very 
few reduction in number of survivals the same exposure time application as ( $\leq 10 \%$ of the whole population) after shown in (fig.3).

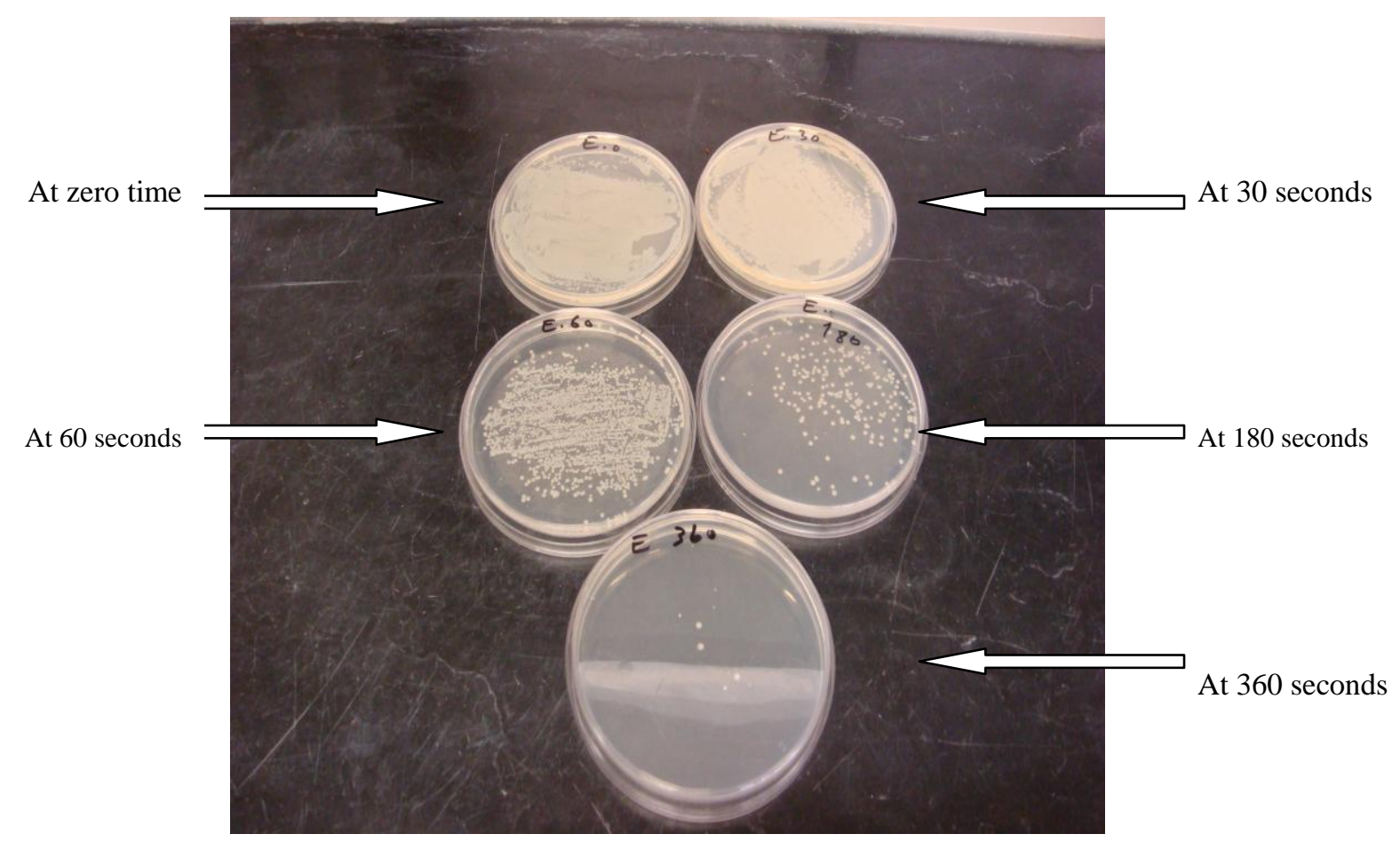

Fig. 2: Effect of plasma on Escherichia coli growth at different time intervals.

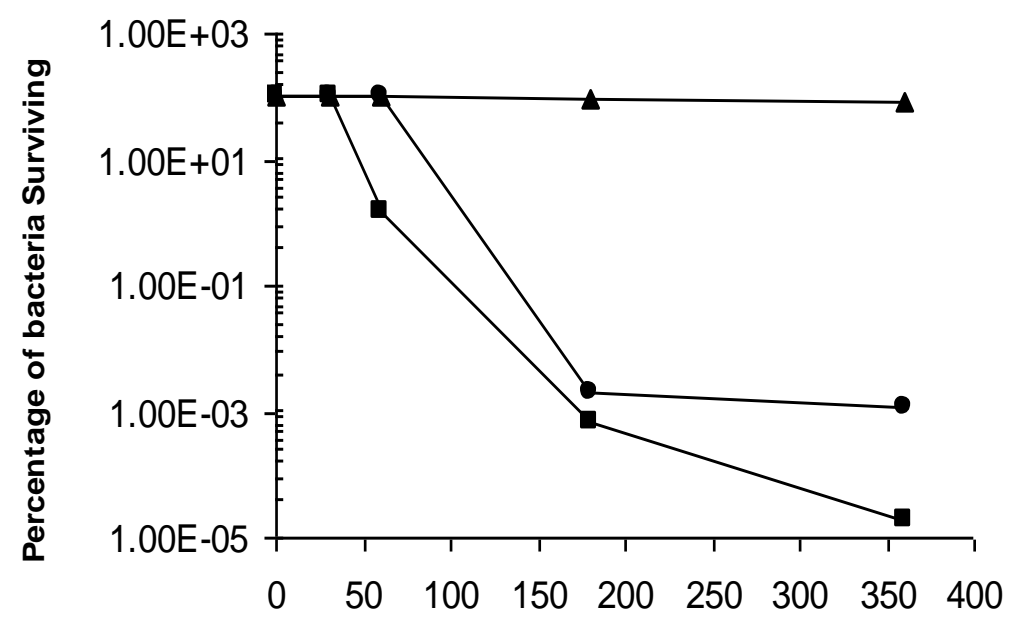

Time (Sec)

Fig. 3: Bactericidal effects of atmospheric cold plasma against $\boldsymbol{\Lambda}$, Pseudomonas aeruginosa $\boldsymbol{\square}$, Esherichia coli and $\bullet$, Staphylococcus aureus. Survival was based on a concentration of approximately $5 \times 10^{6} \mathrm{cfu}^{-\mathrm{ml}^{-1}}$. Cells were collected from stationary phase peptone water cultures.

\section{CONCLUSION}

We can report that the atmospheric cold plasma generated by this method has antibacterial activity against various bacterial strains studied which is in agreement with other studies done by the use of plasma generated by other methods as reported by Kayes et al. 2007.

Atmospheric cold plasma has proven sterilization (kill) capability against both gram-positive and gramnegative bacteria in different extents 
depending on special strain characteristics.

\section{ACKNOWLEDGMENT}

Thanks to all the workers in the plasma research laboratory for

\section{REFERENCES}

Herman, V. B., (1982). Plasma Science and Technology, Cornell University Press, London, p. 37.

Holy, C. E., Cheng, C., Davies, J. E. and Shoichet, M. S., (2001). Optimizing the sterilization of PLGA scaffolds for use in tissue engineering. Biomaterials, 22:25-31.

Kayes, M._M., Critzer, F. J., Wintenberg, K. K., Roth, J. R., Thomas, C. M. and Golden, D. A. (2007). Inactivation of food born pathogens using a one atmosphere uniform glow discharge plasma. Food Born Pathogens and Disease. . 4 (1): 5056.

Kwarteng, K. B. and Stark, C., (1990). Carbon fiber reinforced PEEK_(APC2/AS4) composites for orthopaedic implants. Sampe._Quart., 22: 10-14.

Lerouge, S., Guignot, C., Tabrizian, M., Ferrier, D., Yagoubi, N._and Yahia, L., (2000). Plasma-based sterilization: Effect on surface and bulk properties and hydrolytic stability of reprocessed PU electrophysiology catheters. $J$. Biomed. Mat. Res., 52: 774-782. their help in making this work possible as they offer us the information, place, the materials and instruments to work with.

Moreira, A. J., Mansano, R. D., Andreoli Pinto, T. J., Ruas, R., Luis_da Silva, Z. L., da Silva, M. V. and Verdonck, P. B., (2004). Sterilization by oxygen plasma. Appl. Surf. Sci., 235: 151-155.

Ohkawa, H., Akitsu, T., Tsuji, M., Kimura, H., Kogoma, M. and Fukushima, K., (2006). Pulsemodulated, high frequency plasma sterilization_at atmospheric pressure. Surf. Coat. Technol. 200: 5829-5835.

Pérez-Martínez, J. A. et al. (2007). An RF micro plasma facility development for medical applications. Surf. Coat. Technol., 201: 5684-5687.

Stavridi, M.; Katsikogianni, M. and Missirlis, Y. F. (2003). The influence of surface patterning / sterilization on the haemocompatibility of polycaprolactones. Mater. Sci. Eng., 23: 359-365.

Tortora J. G., Funke B. R. and Case C. L., $10^{\text {th }}$ edition (2010). Microbiology an introduction, Chapter 7:185-207. Pearson Education, Inc.,publishing as Pearson Benjamin Cummings. 